\title{
Electro-optic analysis of the influence of target geometry on electromagnetic pulses generated by petawatt laser-matter interactions
}

\author{
Timothy Robinson ${ }^{1, *}$, Samuel Giltrap ${ }^{1}$, Samuel Eardley ${ }^{1}$, Fabrizio Consoli ${ }^{2}$, Riccardo De Angelis ${ }^{2}$, Francesco Ingenito ${ }^{2}$, \\ Nicholas Stuart ${ }^{1}$, Claudio Verona ${ }^{3}$ and Roland $A$ Smith $^{1}$ \\ 1 Blackett Laboratory, Imperial College London, Prince Consort Road, London SW7 2AZ, United Kingdom \\ 2 ENEA - C.R. Frascati, Dipartimento FSN, Via E. Fermi 45, 00044 Frascati, Italy \\ 3 Dipartimento di Ingegneria Industriale, Università di Roma Tor Vergata, Via del Politecnico 1, I-00133 Roma, Italy
}

\begin{abstract}
We present an analysis of strong laser-driven electromagnetic pulses using novel electro-optic diagnostic techniques. A range of targets were considered, including thin plastic foils $(20-550 \mathrm{~nm})$ and mass-limited, optically-levitated micro-targets. Results from foils indicate a dependence of EMP on target thickness, with larger peak electric fields observed with thinner targets. Spectral analysis suggests high repeatability between shots, with identified spectral features consistently detected with $<1 \mathrm{MHz}$ standard deviations of the peak position. This deviation is reduced for shots taken on the same day, suggesting that local conditions, such as movement of metal objects within the target chamber, are more likely to lead to minor spectral modifications, highlighting the role of the local environment in determining the details of EMP production. Levitated targets are electrically isolated from their environment, hence these targets should be unable to draw a neutralization current from the earth following ejection of hot electrons from the plasma, in contrast to predictions for pin-mounted foils in the Poyé EMP generation model. With levitated targets, no EMP was measurable above the noise threshold of any diagnostic, despite observation of protons accelerated to $>30 \mathrm{MeV}$ energies, suggesting the discharge current contribution to EMP is dominant.
\end{abstract}

\section{Introduction}

Intense electromagnetic pulses (EMP) are often observed alongside high-intensity laser-matter interactions [1-9], such as those studied in petawatt-class laser facilities [10]. These transient radiofrequency (RF) fields can be problematic for experiments due to their coupling to electronic devices in the immediate vicinity, with the potential to damage valuable equipment or result in data losses from electronic diagnostics, such as CCD cameras. Despite these issues, EMP can be used as a complementary diagnostic, and as a source of intense fields for radiation-hardening tests or electromagnetic compatibility applications.

During intense laser-plasma interactions, hot electrons are ejected from the target, resulting in a netpositively charged plasma. EMP generation is typically attributed to two complementary models: firstly, the ejected electrons strike the metal walls of the target chamber, acting as a charge stimulus which excites the electromagnetic resonant cavity modes [10]. The second (Poyé) model relates to the dynamic target charging leading to a discharge current of cold electrons drawn through the target support assembly to maintain quasineutrality; this transient current results in antenna-like emission from the target holder [11,12]. However, target geometry's role in EMP generation has not been extensively studied. Poyé describes a dependence on the surface area of planar targets due to lowering of the potential barrier experienced by electrons as charge redistributes across the surface [11], but there are no published results using "unconventional" targets such as ultra-thin plastic foils or mass-limited isolated spheres. In this work carried out at Vulcan Petawatt and the Cerberus laser system at Imperial College (an OPCPA system operating at $1054 \mathrm{~nm}$ ), we consider the effects on EMP of varying the thickness of plastic foil targets, and using levitated micro-targets trapped in-vacuo using an optical levitation trap developed by Price et al [13].

Electro-optic (EO) probing techniques are inherently low-noise, as signal transport is achieved via optical fiber-link to which ambient EMP fields do not couple. Furthermore, they provide absolute measurements of electric fields via the longitudinal Pockels effect in optical crystals, where polarization changes scale linearly with the electric field in a crystal [14]. Hence, no numerical integration is required to determine the fields, such that constant or low-frequency field components are recovered. The data presented in this article was obtained using the EO diagnostic described in detail in a separate publication [15], and a more compact version during Cerberus experiments, described in Section 2.

*orresponding author: timothy.robinson10@imperial.ac.uk 


\section{Experimental Architecture}

The diagnostic setups deployed for EMP measurement during the Vulcan and Cerberus experiments studying laser-interactions with novel targets are shown in Fig. 1.

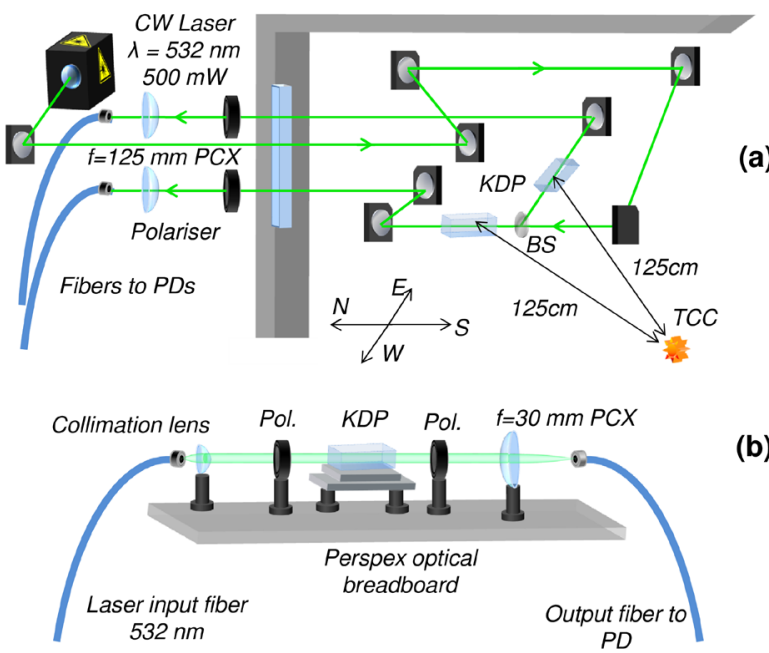

Fig. 1: The prototype and second generation electro-optic EMP diagnostics used for experiments at (a) Vulcan PW (adapted from [15]), using a free-space setup with the laser split between two KDP probes mounted on metal breadboards using a beam-splitter (BS), and (b) using the Cerberus laser.

At Vulcan, the probe KDP crystals were oriented to have their longitudinal axes aligned with the Vulcan target chamber North-South (N/S) and East-West (E/W) directions, and were located $1.25 \mathrm{~m}$ from target chamber centre (TCC), with no direct line of sight to the target; this setup is described in greater detail in [15]. For the Cerberus experiment, a more compact all-dielectric single channel diagnostic with fiber beam transport into and out of the chamber was used, such that no loss of optical alignment occurred upon moving the diagnostic or pumping down the chamber to low pressures.

As the Cerberus laser energies on-target were considerably less than at Vulcan $(\sim 1-2 \mathrm{~J}$ at $\sim 650$ fs instead of $\sim 300-400 \mathrm{~J}$, both focused to $\sim 5 \mu \mathrm{m}$ FWHM spots), the KDP was placed within the $1 \mathrm{~m}$ diameter steel spherical chamber, $0.25 \mathrm{~m}$ from the target, where the EMP fields radiated from the target assembly are expected to be stronger according to a $1 / r^{2}$ scaling. The sensor crystals were shielded by $5 \mathrm{~mm}$ of plastic, with opaque tubes enclosing the beam path to minimize stray light collection by the output fiber-coupling optics.

\section{Time-domain electric field behaviour}

\subsection{Foil targets}

Key shot and target information relating to the ultra-thin plastic targets used during the Vulcan experiment can be found in Table 1. The Vulcan results give some indication that the peak EMP fields generated during the interaction lightly scale with the target thickness, with a trend towards peak fields increasing with decreasing thicknesses, as seen in Fig. 2. Only the dominant N/S mode results are shown, as the weaker E/W results suffer from signal-to-noise issues, making interpretation difficult. Conversely, the drive pulse energies on target do not seem to strongly affect the peak E-fields detected by the EO diagnostic, at least within the small energy range and target thicknesses considered, shown in Fig. 3.

Table 1. Shot and target information for shots with EMP data from Vulcan PW, where $T$ is target thickness, $U_{t a r}$ is energy on target, $I_{0}$ is peak intensity and $\Delta t$ is the FWHM pulse duration.

\begin{tabular}{|c|c|c|c|c|c|}
\hline $\begin{array}{c}\text { Shot } \\
\#\end{array}$ & Material & $\begin{array}{c}\boldsymbol{T} \\
{[\mathbf{n m}]}\end{array}$ & $\begin{array}{c}\Delta \boldsymbol{t} \\
{[\mathbf{p s}]}\end{array}$ & $\boldsymbol{U}{ }_{\text {tar }}[\mathbf{J}]$ & $\begin{array}{c}\boldsymbol{I}_{o} \\
{\left[\mathbf{1 0}^{20} \mathbf{W} \mathbf{c m}^{2}\right]}\end{array}$ \\
\hline 14 & Parylene-N & 550 & 1.00 & 372 & 14.3 \\
18 & Parylene-N & 269 & 1.10 & 369 & 12.4 \\
19 & Formvar & 20 & 1.30 & 341 & 9.7 \\
20 & Parylene-N & 550 & 0.98 & 359 & 14.3 \\
25 & Parylene-N & 550 & 1.00 & 319 & 10.9 \\
29 & Parylene-N & 269 & 1.70 & 386 & 4.8 \\
\hline
\end{tabular}

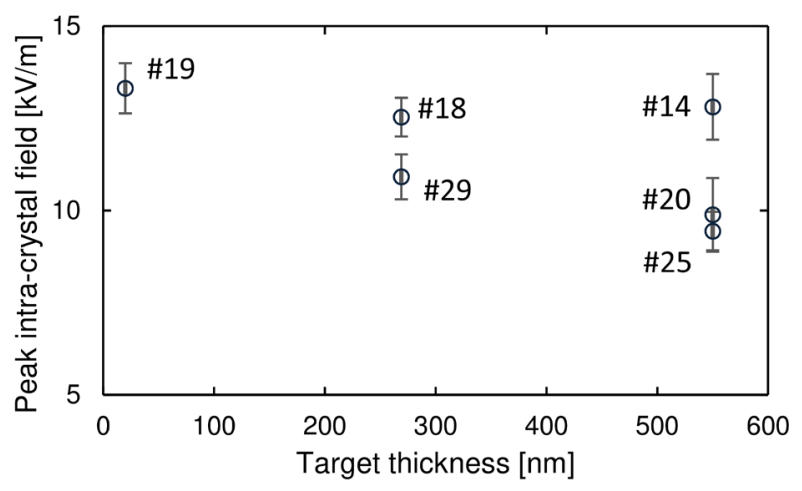

Fig. 2: Variation of the peak-intra crystal field detected by the longitudinal EO diagnostic along the Vulcan N/S direction with the target thicknesses. Shot numbers are given as annotations. The error bars are determined by the noise level on a given shot (annotated), where we have defined error bar lengths by the apparent E-field obtained by processing the reference noise channel data as if it were an E-field measured using EO probes.

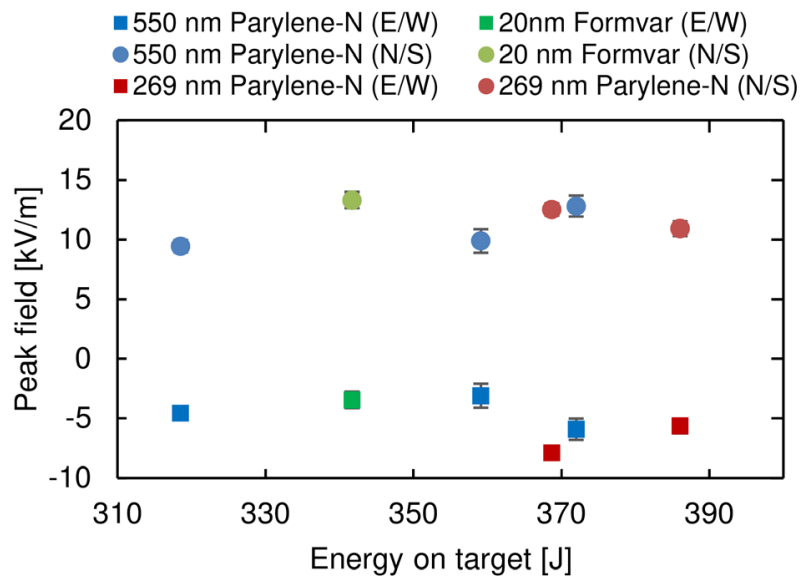

Fig. 3: Variation of the peak-intra crystal field detected by the EO diagnostic along the Vulcan N/S direction with the drive laser pulse energy on target. The error bars are found in the same way as in Fig. 2. 
Although the dataset is small and thus care must be made when drawing conclusions, this data-set represents the first potential experimental observation of the phenomenon of enhanced EMP with thinner foil targets. This observation might be explained by a limited version of enhanced electron heating that occurring in the relativistic regime when the electron skin-depth (as seen by the drive laser) is comparable to the target thickness; even in a classically over-dense plasma, where fields do not propagate, the laser field still exerts a ponderomotive drive on the electrons within the target plasma, resulting in rapid and efficient heating of electrons. These effects have been observed for ultra-thin targets in both simulations and experiment by Yin and Albright, during investigations of ion acceleration in the relativistic transparency or laser break-out afterburner (BOA) regimes [16-18], although the pulse temporal contrast at Vulcan $\left(\sim 10^{6}\right.$ over $\left.200 \mathrm{ps}\right)$ is too low to properly access these regimes. No EMP was detected from Al-foil targets with Cerberus shots - only optical scatter or selfemission coupling into the output fiber was measured suggesting that much weaker EMP fields are generated by joule-level drive pulses.

During the experiment, the E-field detection threshold of the EO-EMP diagnostic was in the range of $\approx 400-700 \mathrm{~V} / \mathrm{m}$, depending on the base voltage level above noise on the photodiode for a given shot. This threshold is approximately half that achieved during the Vulcan experiment, due to the mean zero-field signal on the photodiodes being $\approx 50 \mathrm{mV}$ instead of $\approx 20 \mathrm{mV}$. Hence, the Cerberus EO data can only tell us that the electric field component of the EMP from levitated target or solid target shots driven by few-TW laser pulses (focused to $\approx 5-10 \mu \mathrm{m}$ spot diameters) is less than $\approx 500 \mathrm{~V} / \mathrm{m}$, despite the probe being placed $20 \mathrm{~cm}$ from TCC, compared to $1.25 \mathrm{~m}$ while at Vulcan PW. This highlights the relative insensitivity of EO diagnostics in comparison to conductive probes, although strategies for improving this are outlined in [15].

As the EO probe is mobile, measurements were attempted along and perpendicularly to the interaction chamber's radial axis, as well as at $180^{\circ}$ to potentially reduce the amount of incident scattered light, in order to determine if peaks were self-emission or real fields. The distance of the probe crystal from TCC was kept fixed. This means that the radiated electric field strengths were less than $\approx 500 \mathrm{~V} / \mathrm{m}$ in all probing directions, which is still a relatively large value. As an additional check, the Faraday cage containing the oscilloscope was left open for some shots to look for ambient electrical noise; none was detected on the oscilloscope. This suggests that there is very little EMP emission from the few-joule laser-matter interactions achievable with Cerberus.

\subsection{Optically levitated micro-targets}

According to the Poyé model, the return current drawn through the target assembly is a key contributor to EMP generated in a laser-plasma interaction. Hence, a target geometry which is completely electrically isolated from the interaction chamber and earth should remove the antenna-like RF radiation component from the target holder, and consequently reduce the EMP. Optically levitating targets is a practical method for achieving these conditions. At Vulcan, $\approx 10 \mu \mathrm{m}$ silicone oil droplets were used due to their in-vacuo stability. Further development of the optical trapping technology by S. Giltrap has since enabled hollow spherical shell targets to be trapped, alongside oil droplets and glass spheres; these were also shot during the Cerberus campaign.

The EO diagnostic was unable to detect any EMP, above the minimum resolvable field-strength limit set by experimental noise, from interactions with levitated micro-targets using either Cerberus at the few $\mathrm{J}$ level, or more significantly, Vulcan PW at $>300 \mathrm{~J}$, where accelerated protons of energies $>30 \mathrm{MeV}$ were observed from droplets with no accompanying EMP; the ion acceleration with EMP reduction results will be discussed separately in an upcoming publication led by S. Giltrap. Hence, any generated EMP fields were below the experimental electrical noise level, meaning any fields were less than $\approx 1 \mathrm{kV} / \mathrm{m}$ and $\approx 500 \mathrm{~V} / \mathrm{m}$ during the Vulcan and Cerberus campaigns respectively, and are thus not a significant concern for electrical interference with laboratory equipment. Another upcoming article, led by F. Consoli, will separately cover the comparison between a suite of different EMP diagnostics.

\section{Spectral analysis of EMP}

EMP spectral information can be obtained by Fast Fourier Transform of the time-domain data; an example spectrum (shot \#29) and the ambient EMP noise spectrum from an identical adjacent photodiode and fiber-coupling system are shown in Fig. 4, with the horizontal-plane characteristic modes of the Vulcan PW interaction chamber (without in-chamber metallic hardware), calculated by Mead et al. [10] is indicated by the dotted lines at 76, 101, 152, 202 and $228 \mathrm{MHz}$.

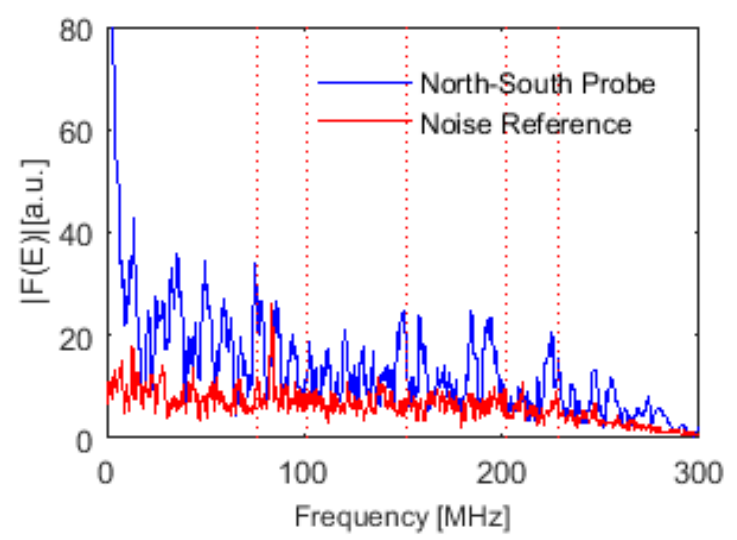

Fig. 4: An example EMP spectrum from shot $\# 29$ of the Vulcan PW experiment with shot and target parameters given in Table 1 . The characteristic chamber cavity modes predicted by Mead for Vulcan PW [10] are indicated by the dotted lines. The reference channel spectrum, using an identical photodiode and fiber-coupling system, is shown to demonstrate the contribution of ambient EMP coupling to the read-out system. 
From this, it is clear that the measured peaks are detected by the probe, with the exception of the $82 \mathrm{MHz}$ feature, corresponding to the vertical fundamental mode of the Vulcan interaction chamber and the $\approx 140 \mathrm{MHz}$ feature. The analysis in the following sections will examine the detailed shot-to-shot reproducibility and variation of spectral measurements with experimental parameters.

\subsection{Reproducibility of spectral features}

The reproducibility of the Vulcan EMP measurements shot-to-shot is important to quantify when deploying novel diagnostics. While there are indeed some differences in the spectra, numerous common spectral features, summarized in Table 2, are observable on all shots, irrespective of target or drive laser pulse parameters. Spectral components up to $200 \mathrm{MHz}$ are shown, as above this frequency the signal-to-noise ratios were too small for meaningful conclusions to be made. Certain components, such as the chamber fundamental modes, are expected to be measured on every shot as the bulk in-chamber architecture (optical tables etc) remains fixed. As previously discussed, other variations in the EMP's spectral content can be attributed to the movement of conductive material such as optical mounts in the chamber between shots, which was not specifically controlled during the experiment. Within the diagnostic's bandwidth range $(0-250 \mathrm{MHz})$, the spectral resolution is limited to $\sim 0.21 \mathrm{MHz}$, in this case by the oscilloscope time-base settings and sampling frequency.

Table 2: Centre frequencies of the peaks measured on a given shot near the identified spectral features given in Column 1.

\begin{tabular}{|c|c|c|c|c|c|c|}
\hline \multirow{2}{*}{$\begin{array}{c}\text { Peak } \\
{[\mathrm{MHz}]:}\end{array}$} & \multicolumn{5}{|c|}{ Observed frequency [MHz] at shot \# } \\
\cline { 2 - 7 } & $\mathbf{\# 1 4}$ & $\mathbf{\# 1 8}$ & $\mathbf{\# 1 9}$ & $\mathbf{\# 2 0}$ & $\mathbf{\# 2 5}$ & $\mathbf{\# 2 9}$ \\
\hline $\mathbf{3 0}$ & 30.2 & 32.5 & 31.0 & 30.8 & 29.4 & 32.3 \\
$\mathbf{4 0}$ & 39.8 & 39.4 & 39.0 & 41.0 & 40 & 41.0 \\
$\mathbf{5 0}$ & 50 & 49.0 & 50 & 51.9 & 49.8 & 49.6 \\
$\mathbf{6 0}$ & 60 & 61.3 & 61.3 & 61.3 & 60.6 & 59.6 \\
$\mathbf{7 1}$ & 72 & 70.2 & 71.6 & 70.8 & 71.3 & 69.6 \\
$\mathbf{7 4}$ & 74.0 & 74.6 & 74.6 & 74.2 & 74.6 & 75.0 \\
$\mathbf{7 6}$ & 75.4 & 76.7 & 78 & 76.7 & 75.8 & 75.0 \\
$\mathbf{9 4}$ & 94.2 & 95.8 & 93.3 & 94.0 & 95 & 94.0 \\
$\mathbf{1 0 2}$ & 102.9 & 100.4 & 102.3 & 102.5 & 104.6 & 102.7 \\
$\mathbf{1 2 0}$ & 120.2 & 120.2 & 121.9 & 119.4 & 120.2 & 120.2 \\
$\mathbf{1 5 2}$ & 151.5 & 152.1 & 152.7 & 152.3 & 152.3 & 150.4 \\
$\mathbf{2 0 0}$ & 199.6 & 199.6 & 199.6 & 199.8 & 199.8 & 199.6 \\
\hline
\end{tabular}

A higher degree of reproducibility between shots taken on the same day of the experiment can be seen in the spectra from shots $\# 18$, \#19 and \#20 of the Vulcan campaign. The other 3 shots, (\#14, \#25 and \#29) all took place on different days during the experiment. The reproducibility is quantified by considering the standard deviation (SD) in the mean center-frequency $\sigma / \sqrt{ } n$ of features measured for a given identified frequency value (Column 1 of Table 2), where we have divided by $\sqrt{n}$ to account for differing sample sizes. The SD for the set of shots restricted to the single day $S_{r s}$, was then subtracted from the SD all the shots, $S_{\text {all }}$, and the differences plotted in Fig. 5. A positive difference value indicates that the $\mathrm{SD}$ in the mean peak position for an identified spectral feature is smaller for the restricted dataset; a majority $(8$ of the 12) features, exhibit a value $\geq 0$, suggesting a higher degree of spectral reproducibility between shots taken on the same day.

This chronological link, with no clear trend linked to target or shot parameters, suggests that minor spectral modifications are more likely to be linked to movement of conductive objects within the interaction chamber between shots on different days, which can affect the frequency modes supported within the chamber [3]. Although it was not monitored and thus cannot be properly confirmed, there is a greater likelihood that the positions of metal objects within the chamber were more similar for shots taken on the same day. While the dataset, and hence sample size, is small such that is difficult to draw reliable conclusions, this evidence suggests the internal chamber architecture and contents are a dominant factor in determining the spectral makeup of the EMP, rather than the thickness of the plastic foil targets used.

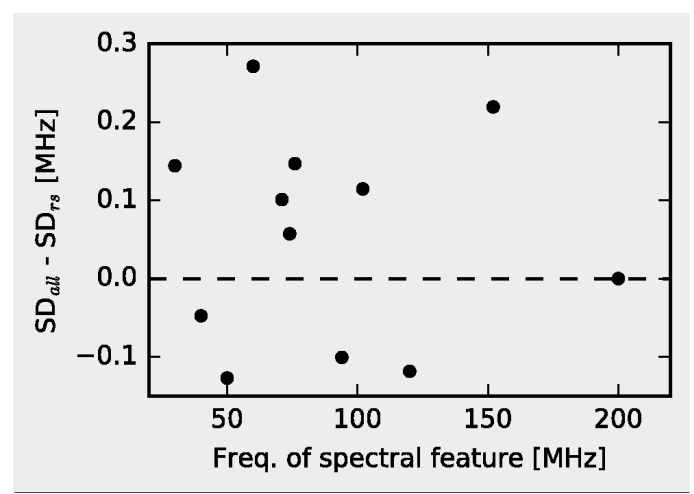

Fig. 5: A plot of the difference between the standard deviations (SD) $S_{\text {all }}$ of the mean position of spectral features between all shots and the SD $S r_{s}$ calculated from a restricted population of shots taken on the same day (\#18,\#19, \#20). The $\mathrm{SD}$ of the mean $\sigma / \sqrt{ } n$, where $\mathrm{n}$ is the number of samples, has been used to account for the different sample sizes.

This does not imply that target geometry is unimportant, as the plastic foils mainly differed in thickness, and no EMP was observed with levitated targets. Furthermore, the target holder assembly was identical for foil shots; as this forms part of the radiating element according to the Poyé model, it follows that the frequency content of the initial radiated EMP will remain consistent. This lack of spectral dependence on the target thicknesses is in contrast to the peak E-fields measured, which are dependent on the ejection of hot electrons, and hence the plasma electron temperatures.

An interesting feature of the electro-optic EMP data is that some of the fundamental chamber resonant modes were observed in the measurement direction perpendicular to their expected direction; for example, the electric field directions of 76 and $152 \mathrm{MHz}$ modes were predicted to be along the $\mathrm{E} / \mathrm{W}$ chamber direction by Mead et al. [10], rather than N/S. In an electromagnetic 
parallelepiped-shaped hollow cavity, TE and TM cavity modes are allowed. Some of these modes are degenerate, so share resonance frequencies. Moreover, the presence of objects in the chamber can locally change the modal field distribution without changing the resonance frequency remarkably [3].

\subsection{Time-frequency analysis}

Short-time window Fourier analysis techniques can be used to infer information about the dynamical evolution of spectral modes emitted following the laser-plasma interaction. The spectrogram for electric fields measured along the N/S direction is shown in Fig. 6. From this, a late-time, secondary detection of EMP fields with frequencies matching the interaction chamber cavity modes was observed. The most likely explanation for this behaviour is that the two previously discussed mechanisms contributing to EMP generation are temporally offset; an electron bunch ejected from the target plasma will have a finite transit time to the interaction chamber walls, whereas a positively charged target can immediately draw a neutralization current through its supporting mechanical assembly.

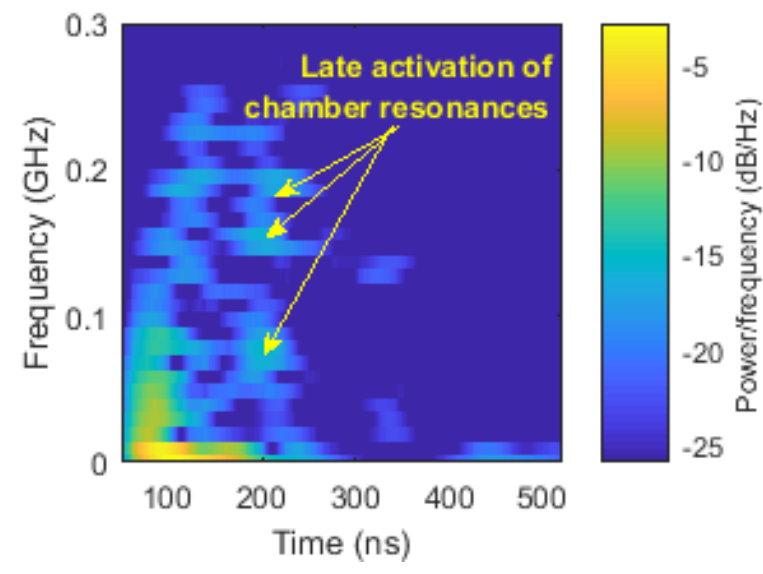

Fig. 6: Time-resolved spectrogram for EMP fields measured along the Vulcan N/S direction taken on shot \#29 (see Table 1). There appears to be some late-time activation of the expected interaction chamber modes, likely due to the ejected electron bunch striking the chamber walls after a finite transit time, whereas EMP radiated due to the discharge current through the target holder can occur as soon as the target becomes positively charged. This plot has been adapted from [15].

This explanation can be tested against time-of-flight (TOF) measurements, sensitive to charged particles and X-rays. We were not able to obtain TOF diagnostic data for the shots with EO-EMP data, however the TOF data from one of the other shots in the series (\#21) is still useful for providing some insight into the delayed activation of the chamber cavity modes. A synthetic monocrystalline CVD diamond detector was used as a TOF diagnostic. This was fabricated by Tor Vergata University laboratories with superficial interdigitalized electrodes $[19,20]$ and operated by colleagues from ENEA-Frascati. It was placed in the Vulcan chamber's western wall, with a direct view of the target and $100 \mathrm{~V}$ biasing, at distance of $\approx 1 \mathrm{~m}$ from TCC.

A TOF trace for shot \#21 is shown in Fig. 7; from this, we can observe the separate arrival of X-rays (at $390 \mathrm{~ns}$ ) as well as "fast" and "slow" particles (above 490 ns) at the diamond detector. As the ambient EMP noise coupling proved problematic to shield, a $60 \mathrm{~m}$ length of RG58 cable was introduced between the detector and the oscilloscope, adding a delay of $\approx 311 \mathrm{~ns}$ and temporally separating the EMP background from the diamond signal.

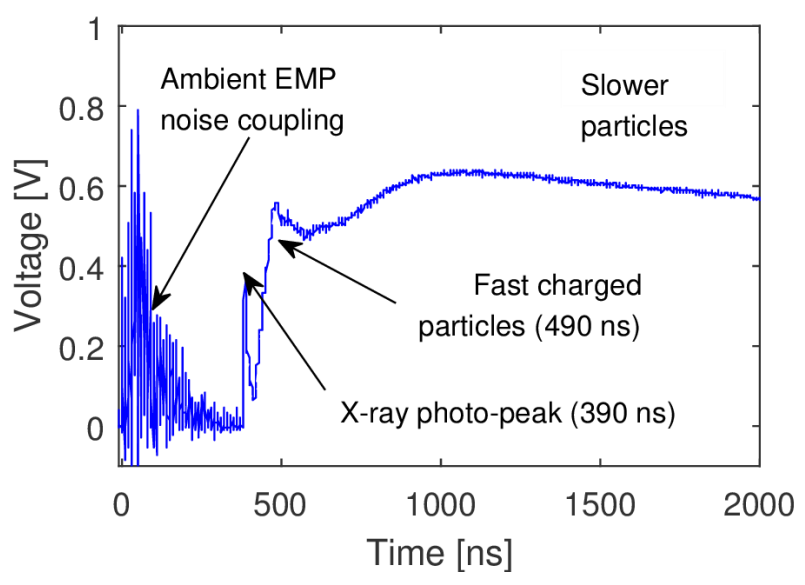

Fig. 7: A time-of-flight trace recorded using a biased CVD diamond detector. A $40 \mathrm{~nm}$ thick Formvar target was used for this shot, with a $1.6 \mathrm{ps}$ FWHM, $357 \mathrm{~J}$ on target, with a peak intensity of $5.6 \times 10^{20} \mathrm{~W} \mathrm{~cm}^{-2}$. The temporal resolution of this measurement was $1 \mathrm{~ns}$. The time axis has been shifted such that the initial laser strike $T_{0}=0$. A $60 \mathrm{~m}$ length of RG58 cable was used to temporally separate the diamond signal from the ambient EMP noise (beginning near $T_{0}=0$ ) coupling to the oscilloscope. The key contributions to the measured signal are labelled on the plot.

The X-ray photo-peak provides a reference for the shifted $T_{0}$, the time of the initial laser strike, relative to other features, as the time taken for prompt photons emitted from TCC to reach the diamond was calculated to $b e \approx 3.3 \mathrm{~ns}$. The arrival of fast particles (few-MeV fast ions, few-keV electrons) peaked $\approx 100$ ns after the photopeak, followed by a broad peak representing slower charged particles. These timescales are approximately consistent with the N/S mode EMP spectrogram for shot \#29 (Fig. 6), where secondary activation of the fundamental chamber modes can be seen $\approx 110$ ns after the initial EMP emission. Although we expect some shot-to-shot variation in the speeds to which ejected fast particles are accelerated, there should be some finite time between fast electrons striking the chamber walls and excitation of RF modes. This provides some evidence for the cavity modes being excited later in time by ejected particles striking the chamber walls, while the radiated fields emitted from the target assembly due to the neutralization current occur earlier in time, closer to $T_{0}$.

If true, this implies that the RF-emission from the target assembly is the larger contribution to EMP in experiments of this type, which could explain the lack of measurable EMP from shots with optically levitated 
micro-droplets, which have no physical discharge current path but still result in charged particle ejection.

\section{Conclusions}

Although the electro-optic EMP dataset obtained from Vulcan PW is limited to only 6 shots, there appear to be certain trends identifiable from the time- and frequency domain data. Firstly, the peak EMP E-fields from ultrathin plastic foil targets appear to negatively correlate with the target thickness; this cannot be explained simply by energies on target being higher for certain shots, as there is no clear correlation between the input energy and the measured peak fields. Hence, at least within the restricted ranges of energy and target thicknesses considered, we suggest that EMP produced by a laserplasma interaction scales with the target geometry, potentially due to enhanced electron heating when the thickness of targets is comparable to the electron skin depth. The lack of measurable EMP from electricallyisolated, optically-levitated, targets also indicates that removing the discharge current component of EMP is very significant in mitigating the EMP problem.

In contrast to the peak intra-crystal field values, the EMP spectra obtained do not appear to show any strong dependence on target thickness, with spectral features largely being reproduced in approximately the same positions on every shot. There appear to be minor shotto-shot variations in the position of several identified spectral features, quantified by the standard deviations of the mean center frequency, which appear to be reduced for shots taken on the same day. This suggests that minor modifications to spectral features are more likely to be driven by movement of conductive objects within the chamber, as opposed to variation in target thicknesses.

Time-frequency analysis combined with time-offlight measurements, which show a late secondary appearance of frequencies matching the resonant cavity modes $\approx 10$ ns after the arrival of fast electrons at the chamber walls, also tentatively suggest that the cavity modes activate later in time than the initial EMP emission (which may include frequency components matching the cavity modes). We thus consider the earlytime EMP contribution to be RF-emission from the target assembly, as in the Poyé discharge current model.

Additional shots at petawatt facilities are required to confirm these observations, however they provide a starting point which can be used to tailor experiments further studies into laser-driven EMP generation, in terms of experimental parameters such as target type and target holder design, particularly as ultra-high energy petawatt systems such as PETAL and Apollon $10 \mathrm{PW}$ become active.

We would like to thank Margaret Notley, our link scientist, for her contributions to planning and running the Vulcan experiment. We acknowledge funding supporting the authors from EPSRC (Studentship Nos 1584149, 1367355, 1228252, 1738157 and Grant No. EP/K022415/1), Industrial CASE awards $(30318116,1103059,30242504,30353497)$ in partnership with AWE plc. The work of F.C and R.D.A. has been partially carried out within the framework of the EUROfusion Consortium and has received funding from the Euratom research and training programme 2014-2018 under grant agreement No. 633053. The views and opinions expressed herein do not necessarily reflect those of the European Commission.

\section{References}

1. C. G. Brown, A. Throop, D. Eder, et al., Journal of Physics: Conference Series (2008), 112, p. 32025.

2. F. Consoli, R. De Angelis, P. Andreoli, et al., 2015 IEEE 15th International Conference on Environment and Electrical Engineering, EEEIC 2015 - Conference Proceedings (2015), pp. 182187.

3. F. Consoli, R. De Angelis, P. Andreoli, et al., Physics Procedia (2015), 62, pp. 11-17.

4. M. De Marco, J. Krása, J. Cikhardt, et al., J. Instrum. 11, C06004-C06004 (2016).

5. M. De Marco, J. Cikhardt, J. Krása, et al., Nukleonika 60, 1-6 (2015).

6. J. L. Dubois, F. Lubrano-Lavaderci, D. Raffestin, et al., Phys. Rev. E - Stat. Nonlinear, Soft Matter Phys. 89, 1-15 (2014).

7. D. C. Eder, R. W. Anderson, D. S. Bailey, et al., Journal of Physics: Conference Series (2010), 244, p. 32018 .

8. J. A. Miragliotta, B. Brawley, C. Sailor, et al., SPIE Defense, Security, and Sensing (2011), 8037, p. 80370N1-8.

9. J. Miragliotta, J. Spicer, B. Brawley, et al., Proc. SPIE 8381, Laser Technology for Defense and Security VIII, 83811N (2012), 8381, p. 83811N1-7.

10. M. J. Mead, D. Neely, J. Gauoin, et al., Rev. Sci. Instrum. 75, 4225-4227 (2004).

11. A. Poyé, S. Hulin, M. Bailly-Grandvaux, et al., Phys. Rev. E - Stat. Nonlinear, Soft Matter Phys. 91, 1-6 (2015).

12. A. Poyé, J. L. Dubois, F. Lubrano-Lavaderci, et al., Phys. Rev. E - Stat. Nonlinear, Soft Matter Phys. 92, 1-17 (2015).

13. C. J. Price, T. D. Donnelly, S. Giltrap, et al., Rev. Sci. Instrum. 86, (2015).

14. G. A. Massey, D. C. Erickson, and R. A. Kadlec, Appl. Opt. 14, 2712-9 (1975).

15. T. S. Robinson, F. Consoli, S. Giltrap, et al., Sci. Rep. 7, 983 (2017).

16. L. Yin, B. J. Albright, B. M. Hegelich, et al., Laser Part. Beams 24, 291-298 (2006).

17. L. Yin, B. J. Albright, B. M. Hegelich, et al., Phys. Plasmas 14, (2007).

18. B. J. Albright, L. Yin, K. J. Bowers, et al., Phys. Plasmas 14, (2007).

19. M. Marinelli, E. Milani, G. Prestopino, et al., Applied Surface Science (2013), 272, pp. 104-108.

20. R. De Angelis, F. Consoli, C. Verona, et al., J. Instrum. 11, C12048-C12048 (2016). 